\title{
Hadronic Physics With Electrons
}

\author{
B. Frois ${ }^{1}$ and J.-F. Mathiot ${ }^{2}$ \\ ${ }^{1}$ Département de Physique Nucléaire, CEN Saclay, Gif-sur-Yvette, France \\ ${ }^{2}$ Division de Physique Théorique, IPN, Orsay, France
}

Key experiments defined in a workshop that discussed strategies for exploring the quark structure of hadrons with electrons beyond $10 \mathrm{GeV}$ emphasize the rôle of the nucleus in providing new and fundamental information.

Detailed long-range plans for nuclear physics have recently been prepared in several European countries. They formed the basis of an in-depth analysis of general trends and priorities showing that unravelling hadronic structure at the fundamental level and establishing its connection to nuclear physics are among the crucial issues [1]. The nature of the strong interaction is understood and there exits in quantum chromodynamics (QCD) a basic theoretical framework for its description. The elementary processes are exchanges of gluons between quarks, the coloured point-like constituents of nucleons. Quarks and gluons cannot be observed as free particles since they are confined in physical particles, the hadrons. The central question is now to understand how nucleons, nuclei and in a more general way all the hadrons are built up from the elementary constituents.

Quark interactions at short distances as described by the lowest order terms of the perturbative development of $\mathrm{QCD}$ are understood. However, long-range nonperturbative effects such as quark confinement, chiral symmetry breaking and the hadronization of quarks are a "black box". Aside from lattice gauge calculations that have well known limitations, one only has crude models with no firm theoretical basis. The situation is unacceptable because a realistic theory of matter will not emerge until one achieves decisive breakthroughs in these problems.

\section{Experimental Hadronic Physics in the 1990's}

The conclusions of the first workshop held two years ago on "Hadronic Physics in the 1990's with Multi-Gev Electrons" [2] emphasized that a high intensity, continuous beam electron accelerator with an energy above $15 \mathrm{GeV}$ represented one of the most appropriate facilities for studying the quark structure of hadrons. The next step was to evaluate in detail the scientific potential of such a facility and the interest it represents for European physicists.

The follow-on workshop to review physics priorities and the ideas developed by various groups in seven European countries was held in Dourdan, France on 8-12
October 1990. Some 200 nuclear and particle physicists took part, including a group of enthousiastic younger scientists whose interests gave birth at the meeting to the Hadronic Physics Network, an association (it already has 60 members) which aims to stimulate cooperation.

The organization of the workshop ensured that the directions for future experiments emerged from a broad consideration of research areas in nuclear structure. There were 12 plenary talks followed by working sessions titled:

- "From Nuclei to Nucleons" (C. Ciofi degli Atti, K. Rith) on the structure of nuclei and the modification of quark distributions in the nuclear medium;

- "From Nucleons to Quarks" (M. Chemtob, P. Mulders, C. Peroni) on the structure and models of the nucleus;

- "From Quarks to Hadrons" (R. Arnold, F. Close, K. van Bibber) on hadronization; - "Electroweak Processes" (R. McKeown, F. Scheck) on parity violation.

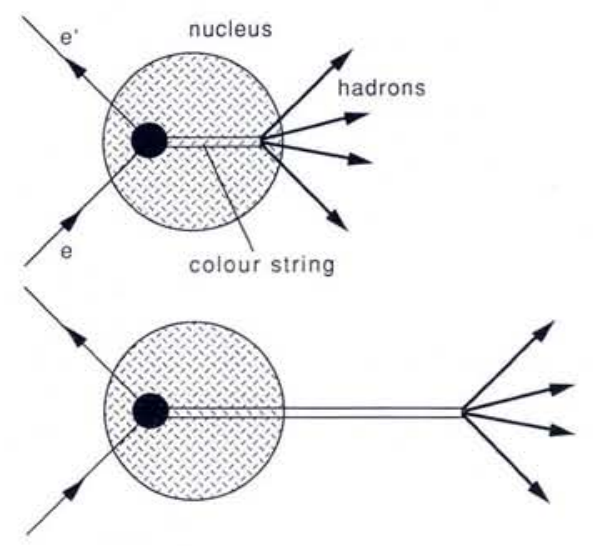

Fig. 1 - Electron-quark interaction: a) upper - production at low and intermediate energies of a colour string that hadronizes in the nuclear medium; b) lower-production at high energy of a string that hadronizes outside the nucleus.

Fig. 2 - Ratio of the attenuations of the production of high energy hadrons in copper and deuterium. Note the abrupt change at energy transfers $v$ between 10 and $30 \mathrm{GeV}$ (from Ref. 3).

\section{Key Experiments}

The strategy that emerged was to devise experiments for studying the evolution of phenomena starting with the well understood perturbative configurations and finishing with the intricacies of confinement. Within this overall framework, three specific topics among a wide range of possible experiments aroused considerable interest:

- Colour transparency: On knocking out a proton from a nucleus by high momentum quasi-elastic scattering one is able to select rare quark configurations of the proton that have a very small size. These almost point-like objects can be considered as miniprotons. As for a wave packet, the miniproton expands, attaining normal size well before the detector is reached. It therefore cannot be observed directly and the trick is to use nuclei as microscopic detectors. A miniproton is much less absorbed by a nucleus than a normal sized proton, an effect that is called colour transparency. So by using different nuclei and by varying the momentum transfer one can probe different quark configurations ranging from the perturbative to the nonperturbative domains.

- Charm production: The regime of charm electroproduction near threshold is of special interest because the differential cross-sections are very sensitive to theoretical hypotheses. The large mass of the charm quark in effect allows precise predictions from QCD of the rate of charm production. The evolution of the $c \bar{c}$ pair in the nuclear medium and the formation of $\mathrm{J} / \psi$ can be studied experimentally using nuclei of different sizes. These studies are essential if we are to find a reliable signature for the deconfinement of quarks in heavy ion collisions.

- Hadronization: Hadronization can be viewed as the breaking of a colour string stretched between two quarks. The rôle of the nucleus as a detector of hadronization at low and intermediate energies is illustrated in Fig. 1a, and at high energy in Fig. 1b. An example of the attenuation of high energy hadron production in copper as compared to deuterium is shown in Fig. 2 (data from SLAC, USA and the European Muon Collaboration, CERN, Geneva).

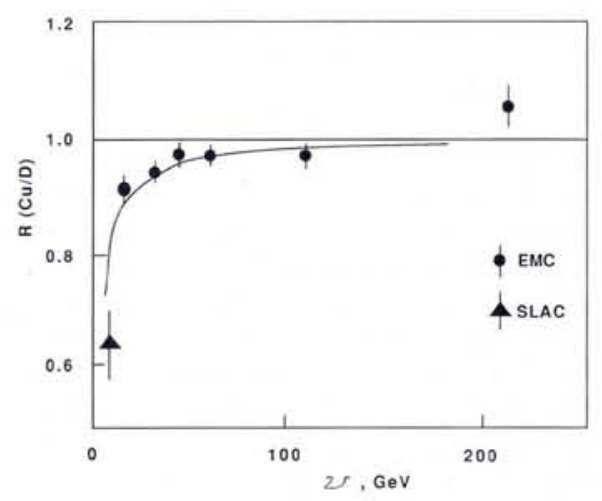


By using nuclei of different sizes together with detection in coincidence of the leading hadron in the final state, it is possible to examine two fundamental aspects of the string, namely the formation length of the hadrons and the string's interaction with the nuclear medium.

\section{An Accelerator for Hadronic Physics}

Electron scattering has been used extensively to study quark distributions since the pioneering work nearly 25 years ago at SLAC by this year's Nobel laureates (see page 208) that gave the first evidence for the presence of quarks in the nucleon. In these experiments, one varies independently the momentum and energy transfer to nuclear constituents by adjusting the incident energy and scattering angle of the electrons. Muons and neutrinos are also used but only to a limited extent as the fluxes that can be achieved in practice are considerably less intense (by at least a factor of $10^{6}$ ).

Their exists today a wealth of electron scattering data but it contains very little information on specific final states. Most of the data that is available on hadronic physics is of poor quality and inconclusive because suitable experimental facilities are lacking. Existing high-energy electron accelerators have pulsed beams, where each beam burst leads to electron-nucleus collisions that produce an extremely large instantaneous flux of all kinds of emitted particles which blinds detectors. In order to observe the coincident emission of particles corresponding to final states, it is imperative to decrease the peak current of each beam burst and to maximize the number of bursts (the ideal being a continuous beam).

An additional requirement is a suitable energy range. The physics programmes that were discussed at the Dourdan workshop invariably require incident electron energies of $15-25 \mathrm{GeV}$. For example, a detailed study of the electroproduction of charmed particles at energies below $15 \mathrm{GeV}$ would be rather limited. Similarly, information about hadronization cannot be revealed using high energy studies above $30 \mathrm{GeV}$, where the production of hadrons would occur outside the nucleus. These and other considerations imply an accelerator having the following characteristics: energy $15-25 \mathrm{GeV}$

$$
\begin{aligned}
& \text { energy resolution }<50 \mathrm{MeV} \\
& \text { duty cycle }>50 \% \\
& \text { intensity }>50 \mu \mathrm{A} .
\end{aligned}
$$

Several possible designs for a continuous intermediate energy electron accelerator were presented in the working session "Experimental Facilities" (P. Dalpiaz, J. Domingo, J.L. Faure). Superconducting RF accelerating cavities will be essential and major improvements in their performance were reported, notably by a group of 60 physicists and engineers at the CEN, Saclay which is pushing the frontiers of this technology and is building a prototype of an electron accelerator using new types of cavity design.

Europe has not yet developed a plan for a facility that would compete with US accelerators, notably the $4 \mathrm{GeV}$ CEBAF electron accelerator under construction and the RHIC relativistic heavy ion collider that is included in the 1991 Federal budget. An European machine with the above characteristics would reach beyond CEBAF: its realization appears challenging but feasible in the light of technical developments that are under study.

Several continuous electron beam facilities will soon be available in Europe (at Bonn, Mainz and Amsterdam) for nuclear structure studies in the 1 to $4 \mathrm{GeV}$ range. Meanwhile, preliminary exploration of hadronic physics will continue with particle physics accelerators such as SLAC and HERA, and with muon beams at Fermilab and CERN. Work at these facilities will show physicists how to optimize new detectors and to design the next generation of experiments.

\section{Future Perspectives}

Now that there is a measure of consensus on the scientific goals and key investigations in hadronic physics, the next step is an in-depth study of the experiments that were proposed at the workshop. The aim will be to arrive at more precise estimates of the optimum characteristics for a state-of-the-art continuous beam electron accelerator. The most obvious concern is to design a cost-effective machine that would satisfy the needs of a large users' community. The conclusions of the workshop will be taken up in the meantime by NuPECC, the Nuclear Physics European Coordination Committee (see Europhysics News 21 (1990) 180) in its preparation, starting next year, of a longterm plan for European nuclear physics.

\section{REFERENCES}

[1] Sick I., Europhys. News 21 (1990) 111. [2] Hadronic Physics in the 1990's with MultiGeV Electrons, Nucl. Phys. A497 (1989) 1.

[3] Bialas A. and Czyzewski J., Phys. Lett. 222B (1989) 132

\title{
Designing With Structural Ceramics
}

\author{
EIW-5, Europhysics Industrial Workshop \\ Petten, 3-6 April 1990
}

The Europhysics Industrial Workshop EIW- 5 was held at Petten in the Netherlands in April 1990. Two cooperating organizations, the Netherlands Energy Research Foundation (ECN) and the Commission of the European Communities, Institute of Advanced Materials, JRC-P, were responsible for the organization.

The meeting aimed to present the stateof-the-art of the application of ceramics in technology, to develop insights into directions for future $R \& D$, and to explore the possibilities for further applications of advanced ceramics in industry. For it is absolutely essential that important progress is made to arrive at a situation where the properties of the materials which can be produced are used to their fullest extent in advanced applications. It must emphasized that designers should demonstrate a greater willingness to use ceramics and to design in such a way that these materials will function satisfactorily. However, a number of critical technologies will also have to be developed:

- Fabrication of dense ceramics and composites without critical defects which degrade the mechanical properties.

- New ways to realize mechanical bonds between ceramics and metals or other ceramics.

- New skills in the field of engineering and design to improve reliability.

Although the workshop intended to focus on the last topic, the presentations and discussion stressed the fabrication and characterization of advanced cera- mics. In this respect, it was found that the aerospace industry is of vital importance to stimulate new developments in structural ceramics. Nevertheless, in spite of this tendency, a number of current industrial applications are worth mentioning to illustrate the present situation.

The aerospace industry clearly focusses on the development of high strength, fibre reinforced composite materials that will be accommodated in a new generation of high velocity aircraft like the STS 2000. The requirements imposed on the materials are well defined, but the materials still find themselves in a state of initial development, with the emphasis on advanced ceramics for locations where material costs are not critical.

A further development worth mentioning is the use of ceramics in the automotive industry. A key feature is to improve high-temperature strength and the reliability. One has not yet succeeded in making components which are sufficiently free from defects to guarantee failure-free operation for long periods of time. This fact gives a strong need for design methods based upon the acceptance of defects in a material (see page 215). Defect tolerance can be obviously achieved by good design involving small volumes, constant wall thicknesses and the absence of tensile stresses or stress concentrations (see page 215). At this moment it should be concluded that practical applications of ceramics in the automotive industry are limited to parts subjected to static stres- 